\title{
Effects of Diet-Supplemented Dimethyl- $\beta$-propiothetin on Growth and Thrust Power of Goldfish, Carp, and Red Sea Bream
}

\author{
Kenji Nakajima* \\ (Received August 3, 1990)
}

\begin{abstract}
Growth and thrust power of goldfish, carp, and red sea bream fed on DMPT-, DMT-, and the vitamin U- supplemented diets were examined. The growth of DMPT, DMT, and the vitamin U group fish in this order was greatly stimulated with increased culture periods. This trend was similarly confirmed in the experiments determining the thrust power of these fish fed on test compounds. During the short experimental period, values of feed efficiency in the DMPT group fish were much higher than those in the other fish group. Experiments examining blood components and viscera weight from tested carp suggest that the marked enhancement of growth and thrust power resulted from the dietary administration of DMPT is closely related to increased or decreased amounts of several blood components in test fish.
\end{abstract}

A tertiary sulfonium compound, dimethyl $\beta$ propiothetin (DMPT), proves to be easily converted to acrylic acid and dimethylsulfide, ${ }^{1,2)}$ the latter of which contributes to the smell we associate with the sea. ${ }^{3,4}$ DMPT further proves to occur mainly in various aquatic organisms. ${ }^{1,5-11}$ ) However, its role in these organisms remained quite obscure, except its role as an osmoprotectant in algae..$^{12,13)}$ We have studied its role in fish, and thus found that among various sulfurcontaining compounds DMPT remarkably stimulates feeding and growth of freshwater and marine fish. ${ }^{14-173}$ Hence, I attempted to examine effects of DMPT on growth and thrust power of freshwater and marine fish in small vessels with dense confinement of test fish, simulating the conditions in aquaculture.

\section{Malterials and Methods}

Dimethylthetin (DMT) (carboxymethyldimethyl sulfonium bromide) and dimethyl- $\beta$-propiothetin (DMPT) (2-carboxyethyldimethyl sulfonium bromide) were synthesized by reacting dimethylsulfide with either bromoacetic acid or 3-bromopropionic acid in equimolar amounts at $42^{\circ} \mathrm{C}$ for 3 or $12 \mathrm{~h}$. Vitamin U (3-amino-3-carboxypropyldimethyl sulfonium chloride) was purchased from Wako Pure Chemicals Co. Ltd., Japan. The crude crystals of DMT and DMPT thus obtained and the vitamin $U$ were washed separately with excess amounts of chilled dry ether, and purified by crystallizing them from their methanol solutions. The structures of synthesized DMT and DMPT were identified by the methods of nuclear magnetic resonance and infrared absorption spectra, melting points etc. Other chemicals were purchased from Wako Pure Chemicals Co. Ltd., Japan.

Goldfish Craccius auratus (six-month age; average weight $1.2 \mathrm{~g}$ ) and carp Cyprinus carpio (one and half-year age; average weight $389 \mathrm{~g}$ ) were purchased from a City Fish Shop in Hyogo Prefecture and the Nango Fish Culture Center in Shiga Prefecture, respectively. Red sea bream Pagrus major (seven-month age; average weight $11.4 \mathrm{~g}$ ) were the kind gift from the Hyogo Prefectural Mariculture, Japan. These fish were reared on a commercial dry pellet in separate acryl containers $(44 \times 90 \times 45 \mathrm{~cm}$ height $)$ containing 150 liters of tap water or the commercial synthetic sea water (Japan Biochemical Co. Ltd.) at $20^{\circ} \mathrm{C}$. Water was aerated and filtered through glass wool with charcoal for the freshwater fish and additionally with a kind of adsorbent (calcium silicate), "Big Ball", (Zensui Co. Ltd., Japan) for red sea bream. The commercial fish diet, "Swimmy mini", used for goldfish and carp was purchased from Nippon Pet Food Co. Ltd., Japan. The commercial diet, "Ajikuranburu", used for the red sea bream was the kind gift from Nisshin Shiryo Co. Ltd., Kobe, Japan.

Effects of DMPT, DMT and vitamin $U$ on growth of goldfish and red sea bream were examined in separate polystylene containers $(12 \times$

* Laboratory of Biochemistry, Department of Nutrition, Koshien University, Momijigaoka, Takarazuka,

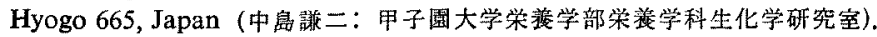


$20 \times 14 \mathrm{~cm}$ height and $16 \times 27 \times 17 \mathrm{~cm}$ height). Ten goldfish and twelve red sea bream were put in each vessel with the water kept at $20^{\circ} \mathrm{C}$, and aerated and filtered through glass wool. The fish were fed on the diet paste freshly prepared from the commercial fish diet powder containing $10 \%$ gluten and either distilled water in the control group or one of the test solutions in the test groups at the ratio of $2: 1(\mathrm{w} / \mathrm{v})$. The test solutions of $1 \mathrm{~mm}$ for goldfish and carp, and of $5 \mathrm{~mm}$ for red sea bream were employed. After being allowed to feed for 20 minutes, goldfish and red sea bream were temporarily moved by net from the containers to another similar one to enable the water to be replaced. Feeding was conducted twice a day (10 a.m. and 4 p.m.). The body weight of goldfish and red sea bream was determined, not individually but en masse, at specified times and the values were expressed as the body weight gain in terms of the percentage of each average weight at the beginning of the experiments. In the trials with eight carp, the experimental conditions were the same as in the goldfish and red sea bream, except that the 150-liter acryl containers were used as test vessels for growth experiments, and that the water after feeding the diet was replaced not immediately but every three days, and then the body weight was individually determined. After the experiments, the carp were anesthetized with MS-222 (ethyl m-aminobenzoate methansulfonate), and the blood was carefully removed from the aorta caudalise by a syringe vertically injected into the abdominal muscle between dorsal and caudal fins. And then the viscera were excised and their weight was determined.

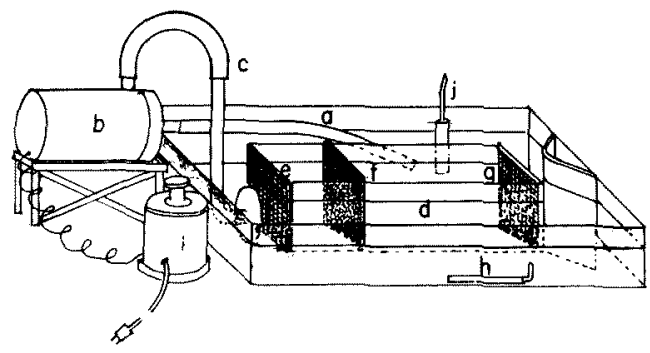

Fig. 1. Schematic diagram of the chamber determining fish thrust power.

The symbol a, suction pipe; b, pump; $c$, introducing pipe; $d$, test chamber; $e, f, g$, lattices; $h$, air sone; $i$, current transformer; $j$, probe of the dissolved oxygen meter.

For experimental conditions see details in the Materials and Methods section.
An apparatus for determining thrust power of test fish is illustrated in Fig. 1: Fresh and sea water $(19 l)$ for goldfish and red sea bream respectively are put in an acryl vessel $(56 \times 35 \times 15$ $\mathrm{cm}$ height). A pump (Iwaki, model MO-30R) is placed outside the vessel, and a suction pipe connected to the pump is extended into the side part of the vessel. An acryl test chamber $(40 \times$ $14 \times 15 \mathrm{~cm}$ height) is disposed in the center part of the vessel as seen in Fig. 1. The suction pipe sucks the water from the side part of the vessel by the operation of the pump, which sends the water through an introducing pipe into the test chamber. Three lattices are disposed at the inlet, fore and outlet of the chamber. The fore lattice is further doubly covered with a square mesh sheet $(4.0 \mathrm{~mm}$ square). These lattices prevent fish from swimming out of the chamber and make water stream uniform everyway in the chamber. Hence, the water circulates in the order of the side part of the vessel, the suction pipe, the pump, the introducing pipe and the test chamber, in the course of which the water flows uniformly everywhere in the chamber and the flow speed is freely changed by a current transformer if necessary. As air stone is provided in the side part of the vessel, so that air can be introduced from an air pipe by the action of an air pump (not shown) and air bubbles can be sent into the water.

Ten goldfish and twelve red sea bream were respectively placed in the test chamber. In a trial, the flow speed was raised little by little by turning a knob on the transformer and adjusted to a constant speed of $34.0 \mathrm{~cm} / \mathrm{s}$ just after $3 \mathrm{~min}$, then the continuous swimming time was recorded until the tails of half the test fish touched the outlet lattice. Experiments determining the swimming time of carp were performed similarly but individually, except that a long acryl vessel $(84 \times$ $29 \times 28 \mathrm{~cm}$ height), a round acryl test chamber $(15 \phi \times 54 \mathrm{~cm})$ and a large pump (Iwaki, Model IP-60) (adjusted to the final flow speed of $44.6 \mathrm{~cm} / \mathrm{s}$ after $3 \mathrm{~min}$ ) were employed.

During the experiments, temperature, $\mathrm{pH}$, and dissolved oxygen in the test water were recorded with a YSI Dissolved Oxygen Meter (Model 57, U.S.A.). The water temperature was kept at $20^{\circ} \mathrm{C}$ and the $\mathrm{pH}$ at around 7.1 with goldfish and carp and 8.2 with red sea bream, while the concentrations of dissolved oxygen changed from 7.7 to $8.2 \mathrm{ppm}$.

Final energy level (calorie) of swimming fish was calculated by determining the drag power ( $g$ 
weight) that is the resistive power to completely sedative fish in a fluid, and then by multiplying it by the following parameters; 980 , the flow speed of the water $(34.0 \mathrm{~cm} / \mathrm{s}$ for goldfish and red sea bream or $44.6 \mathrm{~cm} / \mathrm{s}$ for carp) and each swimming time of the test fish (determined on the 17th, the 16 th and the 27 th day with goldtish, red sea bream, and carp, respectively).

Kits for determining the activities of amylase, transaminases (GOT, GPT) and the amounts of serum total cholesterol, triglyceride, glucose and creatinine were obtained from Wako Pure Chemcals Co. Ltd. The amount of hemoglobin was colorimetrically measured as hematin hydrochloride with a Sahli's Hemoglobinometer (Type M, Erma Optics, Co. Ltd., Japan). The hematocrit valuse (packed cell volume) were determined by the centrifugation method $(10,000 \mathrm{rpm}, 5 \mathrm{~min})$ in a heparinized capillary glass tube (Durmond Scientific Co. Ltd., U.S.A.). Plasma protein level was estimated with a Hitachi Protein Meter (Hitachi Co. Ltd., Japan).

\section{Results}

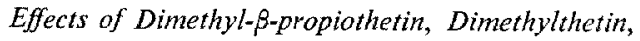
and Vitamin $U$ on Growth and Thrust Power of Goldfish

The effects of diet-supplemented DMPT, DMT and vitamin $U$ on the growth of foldfish were com-

Table 1. Fluctuation of body weight (g) in goldfish and red sea bream

\begin{tabular}{|c|c|c|c|}
\hline \multirow{2}{*}{ Fish/Addition } & \multicolumn{3}{|c|}{ Rearing period (days) } \\
\hline & 0 & 7 & 15 \\
\hline \multicolumn{4}{|l|}{ GOLDFISH } \\
\hline None & $\begin{array}{r}1.57 \\
(0)\end{array}$ & $\begin{array}{l}1.81 \\
\text { (15) }\end{array}$ & $\begin{array}{l}1.75 \\
(12)\end{array}$ \\
\hline DMT & $\begin{array}{r}1.53 \\
(0)\end{array}$ & $\begin{array}{l}1.95 \\
(28)\end{array}$ & $\begin{array}{l}2.10 \\
(37)\end{array}$ \\
\hline DMPT & $\begin{array}{c}1.51 \\
(0)\end{array}$ & $\begin{array}{l}2.11 \\
(40)\end{array}$ & $\begin{array}{c}2.33 \\
(54)\end{array}$ \\
\hline Vitamin $\mathbf{U}$ & $\begin{array}{r}1.55 \\
(0)\end{array}$ & $\begin{array}{l}1.86 \\
(20)\end{array}$ & $\begin{array}{l}1.87 \\
(21)\end{array}$ \\
\hline \multicolumn{4}{|c|}{ RED SEA BREAM } \\
\hline None & $\begin{array}{r}16.5 \\
(0)\end{array}$ & $\begin{array}{c}18.2 \\
(10)\end{array}$ & $\begin{array}{r}20.6 \\
(25)\end{array}$ \\
\hline DMPT & $\begin{array}{c}16.1 \\
(0)\end{array}$ & $\begin{array}{r}29.0 \\
(80)\end{array}$ & $\begin{array}{r}31.5 \\
(96)\end{array}$ \\
\hline Vitamin $\mathrm{U}$ & 16.3 & $\begin{array}{r}19.6 \\
(20)\end{array}$ & $\begin{array}{r}19.6 \\
(20)\end{array}$ \\
\hline $\begin{array}{l}\text { Values are shown a } \\
\text { twelve red sea bream. } \\
\text { Numbers in parent? } \\
\text { the corresponding start } \\
\text { For experimental co } \\
\text { Methods section. }\end{array}$ & body wei & ght of ten & $\begin{array}{l}\text { oldfish and } \\
(\%) \text { against } \\
\text { terials and }\end{array}$ \\
\hline
\end{tabular}

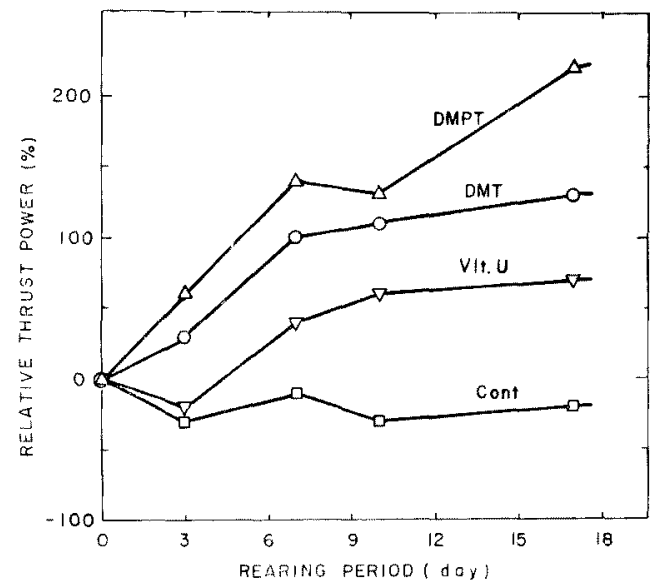

Fig. 2. Effects of dietary dimethyl- $\beta$-propiothetin, vitamin $U$ on thrust power of goldfish.

The average swimming time of the DMPT, the DMT, the vitamin $\mathrm{U}$, and the control groups at the start of the experiments was $4.8,5.5,3.7$, and $4.2 \mathrm{~s}(n=3)$, respectively.

For experimental conditions see details in the Materials and Methods sections.

pared. Results are given in Table 1. The body weight gain was effectively elevated in the order of the DMPT, the DMT, and the vitamin U groups during the experimental period, showing 4.5, 3.1 and 1.8-fold increases against that of the control group on the 15 th day, respectively.

Thrust power of test fish fed on diet-supplemented DMPT, DMT, and vitamin $U$ was examined. The results are presented in Fig. 2, indicating that the thrust power of each was greatly enhanced in the order of the DMPT, the DMT, and the vitamin $U$ groups as opposed to its decrease in the control group.

Effects of Dimethyl- $\beta$-propiothetin and Vitamin $U$ on Growth and Thrust Power of Red Sea Bream

The effects of diet-supplemented DMPT and vitamin $U$ on the growth of red sea bream were compared. The results are presented in Table 1. The body weight in the DMPT group was the most greatly increased, while that in the vitamin $\mathbf{U}$ group was little less than in the control group. The weight gain of the DMPT and the vitamin $U$ groups was 3.8 and 0.8 -fold against that of the control group on the 15 th day, respectively.

Thrust power of red sea bream fed on dietsupplemented DMPT was compared to that of the control group. Results are shown in Fig. 3. The rapid increase of the thrust power in the 


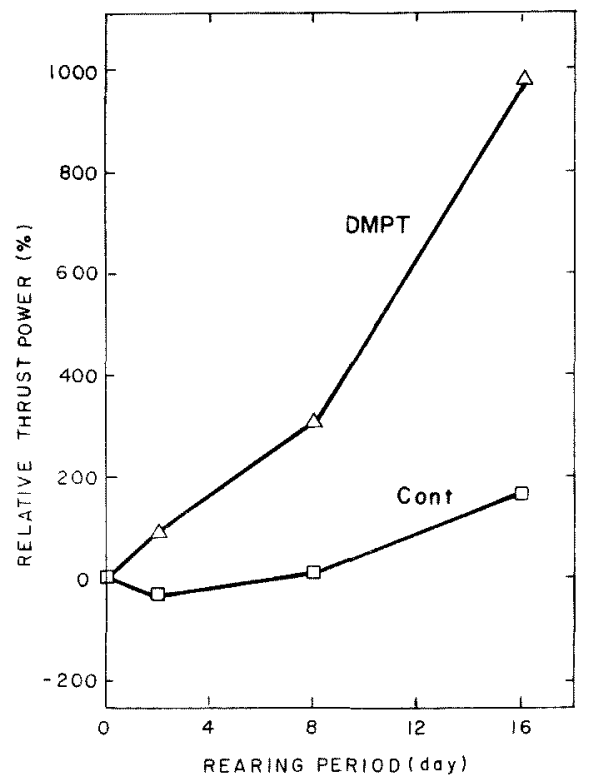

Fig. 3. Effects of dietary dimethyl- $\beta$-propiothetin on thrust power of red sea bream.

The average swimming time of the DMPT and the control groups at the start of the experiments was 3.3 and $3.1 \mathrm{~s}(n=3)$, respectively.

For experimental conditions see details in the Materials and Methods section.

DMPT group was observed against its gradual increase in the control group during the experimental period; the former being 6.5 -fold against the latter on the 16th day.

Growth behaviors of goldfish and red sea bream fed on the DMPT-supplemented diet have been studied in more deteil in the previous papers. ${ }^{16,17)}$

Effects of Dimethyl- $\beta$-propiothetin and Vitamin $U$ on Growth and Thrust Power of Carp

The body weight gain of test fish fed on the dietsupplemented DMPT and vitamin U were compared. The results are presented in Fig. 4, which shows that the weight gain of the DMPT group was the highest, that of the vitamin $U$ group gave a little increase, but that of the control group rather decreased. Effects of diet-supplemented DMPT and vitamin $U$ on thrust power of carp were examined. Results are given in Fig. 5. The thrust power of the DMPT group rapidly increased after the 6th day, while that of the vitamin $\mathrm{U}$ and the control groups continued to decrease till the 12 th day and thereafter reached almost plateaus.

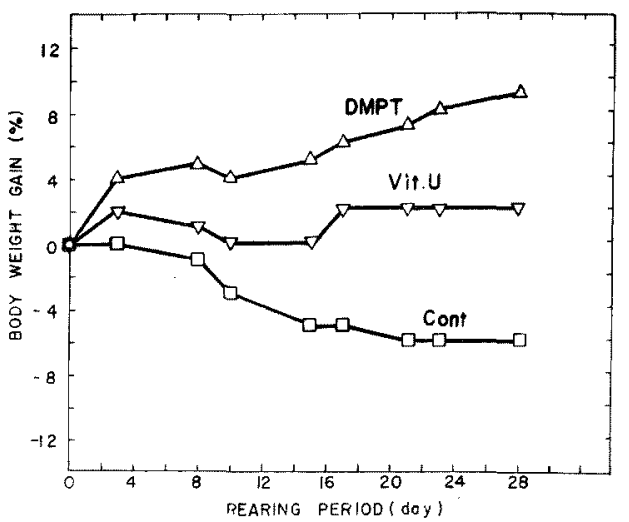

Fig. 4. Effects of dietary dimethyl- $\beta$-propiothetin and vitamin $U$ on growth of carp.

The body weights of the DMPT, the vitamin $\mathrm{U}$ and the control groups at the start of the experiments were $382.4 \pm 32.0,391.4 \pm 32.0$ and $418.3 \pm 35.8 \mathrm{~g}($ mean \pm S.D. $(n=8))$.

For experimental conditions see details in the Materials and Methods section.

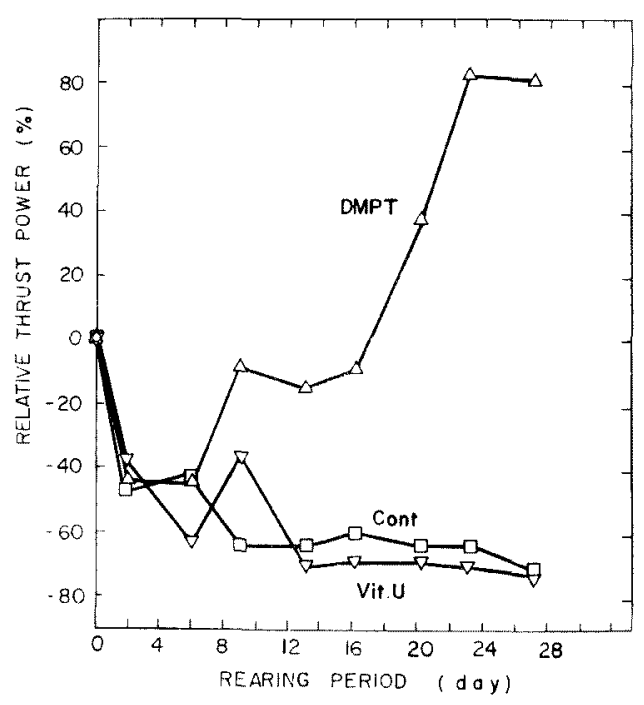

Fig. 5. Effects of dietary dimethyl- $\beta$-propiothetin and vitamin $U$ on thrust power of carp.

The swimming time of the DMPT, the vitamin $\mathbf{U}$, and the control groups at the start of the experiments were $6.5 \pm 1.7,5.7 \pm 3.3$, and $5.8 \pm$ $2.3 \mathrm{~s}$ (mean \pm S.D. $(n=8)$ ), respectively.

For experimental conditions see details in the Materials and Methods section.

Properties of Viscera and Blood from Carp Tested

Properties of viscera and blood from the test carp were examined on the 28 th day. Results are 
Table 2. General profiles of viscera and blood from DMPT and vitamin U-supplemented carp

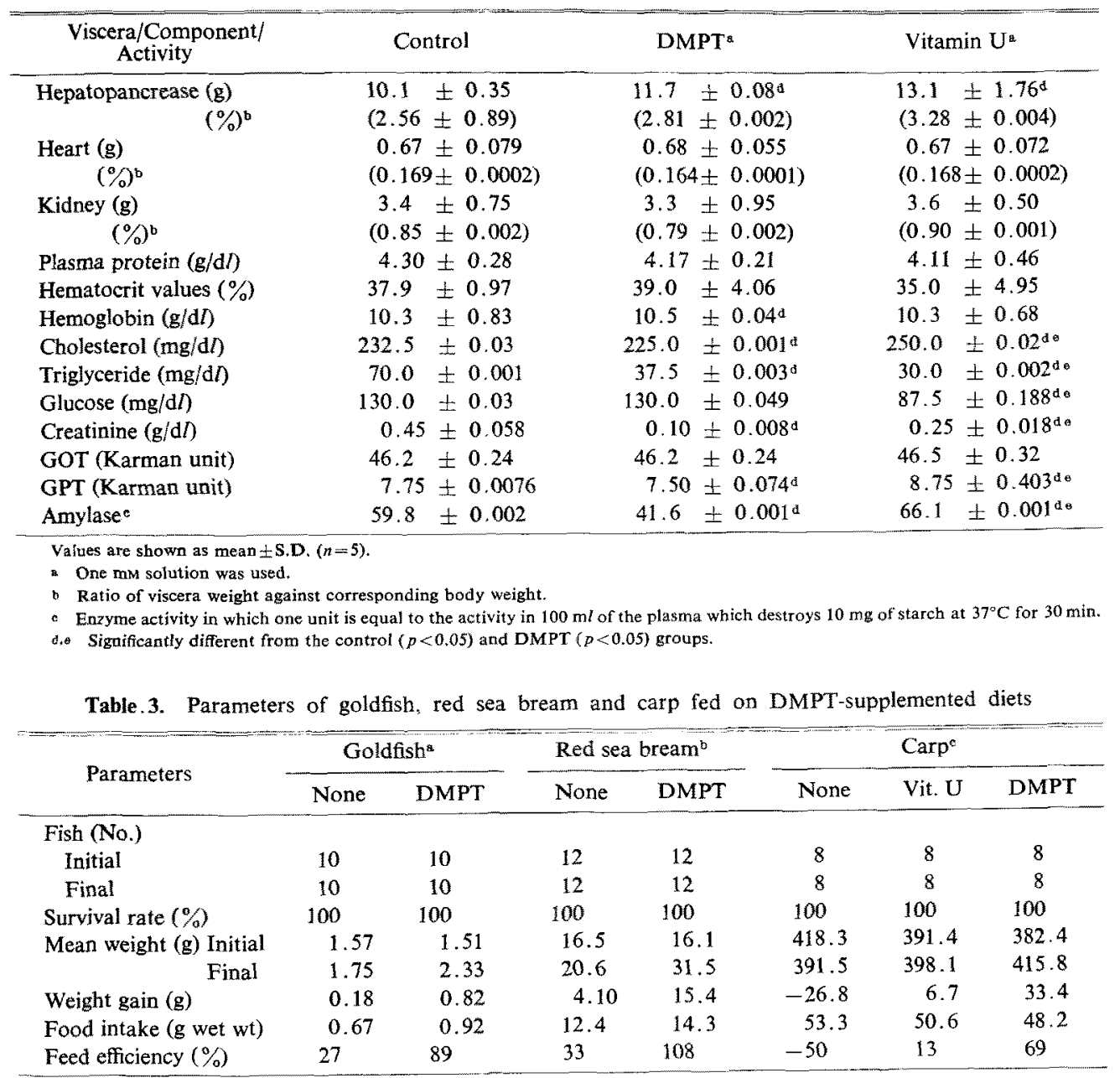

a.b,c, Rearing periods are 15,15 , and 28 days, respectively.

Table 4. Final energy level in thrust power of goldfish, red sea bream and carp

\begin{tabular}{llcc}
\hline Fish & Supplement & $\begin{array}{c}\text { Swimming time } \\
(\mathrm{s})\end{array}$ & $\begin{array}{c}\text { Energy level } \\
(\text { cal })\end{array}$ \\
\hline Goldfish & None & 0.7 & $1.27 \times 10^{-4}$ \\
& Vitamin U & 3.1 & $2.55 \times 10^{-4}$ \\
& DMT & 7.4 & $3.58 \times 10^{-4}$ \\
& DMPT & 9.0 & $4.90 \times 10^{-4}$ \\
Red sea bream & None & 15.0 & $5.37 \times 10^{-3}$ \\
& DMPT & 32.3 & $1.16 \times 10^{-2}$ \\
Carp & None & $-2.7^{\mathrm{a}}$ & $1.59 \times 10^{-2}$ \\
& Vitamin U & $-1.8^{\mathrm{a}}$ & $1.62 \times 10^{-2}$ \\
& DMPT & 3.5 & $5.71 \times 10^{-2}$ \\
\hline
\end{tabular}

a Shows that the talls of half the test fish had touched the outlet lattice before the initiotion $(3 \mathrm{~min}(44.6 \mathrm{~cm} / \mathrm{s}))$ of the $\mathrm{sw}$ imming test (averase time, $2 \mathrm{~min}$ and $57.3 \mathrm{~s}$ (about $44.3 \mathrm{~cm} / \mathrm{s}$ ) with None, $2 \mathrm{~min}$ and $58.2 \mathrm{~s}$ (about $44.6 \mathrm{~cm} / \mathrm{s}$ ) with vitamin $\mathrm{U}$ ). The corresponding energy level was roughly calcuculated, based on the supposition that the flow speed of the water was linearly raised to the final speed of $44.6 \mathrm{~cm} / \mathrm{s}$ with a lapse of $3 \mathrm{~min}$.

For experimental conditions sec details in the Materials and Methods section. 
shown in Table 2. The weight of viscera was almost the same in these experimental groups, except for the higher values of hepatopancreas in the vitamin U group (different from DMPTs $(p<0.1)$ ). The blood of fish in the control group showed the highest values of triglyceride and creatinine, higher values of glucose, and lower values of cholesterol and amylase than those of the other groups. The blood of the fish in the DMPT group gave higher values of glucose, slightly higher values of hemoglobin, lower values of triglyceride, and the lowest values of cholesterol, creatinine, GPT, and amylase. The blood of the fish in the vitamin $U$ group represented the highest values of cholesterol, GPT, and amylase, and the lowest values of triglyceride and glucose.

\section{Several Parameters Obtained from Test Fish}

Results of the survival rate, feed e ciency and energy level of the test fish in the present experiments are shown in Tables 3 and 4 . The values of food intake with goldfish and red sea bream were increased, but those with carp rather decreased by the supplementation of DMPT, probably due to too dense confinement of the carp. Yet, all of the feed efficiencies obtained in the DMPT groups were much higher than those in the control and the vitamin U groups (Table 3). The final energy level was also highest in the DMPT groups. The values of the DMPT, the DMT, and the vitamin $U$ groups in goldfish were 3.9, 2.8, and 2.0-fold, those of the DMPT group in red sea bream were 2,2-fold, and those of the DMPT and the vitamin $U$ groups in carp 3.6 and 1.0-fold against those of corresponding control groups (Table 4).

\section{Discussion}

The effects of diet-supplemented dimethyl- $\beta$ propiothetin (DMPT), dimethylthetin (DMT) and vitamin $U$ on growth and thrust power of goldfish, carp, and red sea bream were examined. Dietsupplemented DMPT proved to effectively stimulate growth of juvenile goldfish and red sea bream, and middle-sized carp, when compared with DMT and/or vitamin $U$, both of which are closely related compounds to DMPT. Similar results have already been obtained for the growth of goldfish, ${ }^{10}$ red sea bream, yellowtail, and flounder fed on a DMPT-supplemented commercial food. ${ }^{17)}$ Present experiments further indicated that dietary administration of DMPT drastically enhances the thrust power of goldfish, carp, and red sea bream. This proves that DMPT can activate not only the feeding and growth, but also movement of freshwater and marine fish. These findings suggest that dimethyl- $\beta$-propiothetine, a tertiary sulfonium compound which possibly originates from algae ${ }^{8, b}$ and higher plants, ${ }^{18,18)}$ has a potential as a new vitaminoid or a hormonal compound effective for various species of fish with varied food habits.

The viscera weight and blood properites of tested carp were also examined. There were almost no differences in the weight of viscera among the experimental groups, except for higher values of hepatopancreas in the vitamin $U$ group. Furthermore, the transaminase (GPT) and amylase activities of not the DMPT group but the vitamin $U$ group gave the highest values. These results suggest that the vitamin $\mathrm{U}$-supplemented diet afforded structurally and functionally bad effects on the hepatopancreas and kidney of the tested carp, but that the DMPT-supplemented diet gave almost no bad effect. Higher level of hemoglobin, the same but large amounts of glucose, lower amounts of cholesterol, and much lower amounts of triglyceride in the DMPT group than those in the control group may well reflect on the marked enhancement of growth and thrust power of the DMPT supplemented carp. Orally administrated DMPT to the rat significantly increased their running and suspending power, which was compatible with increased amounts of serum glucose and hemoglobin. ${ }^{20}$ ) Therefore, the main parameters which elicit stimulative growth and active movement of fish and rat are considered to be increased amounts of serum glucose (energy source) and hemoglobin (oxygen carrier).

Thus, DMPT may be suggested to be rather a hormone (or a hormonal agent) than a vitamin in fish. This possiblity may be strengthened by the new finding that DMPT is biosynthesized by fish themselves. But, it remains obscure at present, although it has been proven to be contained in various fish. ${ }^{\theta-11}$

The high activation effects of DMPT-supplemented commercial diet on thrust power of test fish also suggest that fish fed on DMPT might be very resistive to a number of environmental stresses due to dense confinement of fish, changes in dissolved oxygen level and water temperature, pollution of water, and diseases in aquaculture.

\section{Acknowledgments}

The skillful assistances of Messrs R. Kawase 
and T. Kawamori are gratefully acknowledged.

\section{References}

1) A. Challenger and M. I. Simpson: J. Chem. Soc, 1591-1597 (1948).

2) G. L. Cantoni and D.C. Anderson: J. Biol. Chem., 222, 171-177 (1956).

3) H. Iida, K. Nakamura, and T. Tokunaga: Nippon Suisan Gakkaishi, 51, 1145-1150 (1985).

4) K. Kasahara and K. Nishibori: Nippon Suisan Gakkaishi, 53, 673-676 (1987).

5) Y. Ishida: Mem. Coll. Agr., Kyoto Univ., 94, 47-82 (1968).

6) H. Iida and T. Tokunaga: Nippon Suisan Gakkaishi, 52, 557-563 (1986).

7) T. Tokunaga, H. Iida, and K. Nakamura: Nippon Suisan Gakkaishi, 43, 1209-1217 (1977).

8) R. G. Ackman and J. Hingley: J. Fish. Res. Bd. Can., 25, 267-284 (1968).

9) H. Iida, J. Nakazoe, H. Saito, and T. Tokunaga: Nippon Suisan Gakkaishi, 52, 2155-2161 (1986).

10) R. G. Ackman, J. Hingley, and A. W. May:
J. Fish. Res. Bd. Can., 24, 457-461 (1967).

11) R. G. Ackman, J. Hingley, and T. Maskey: $J$. Fish. Res. Bd. Can., 29, 1085-1088 (1972).

12) R. H. Reed: Mar. Biol. Letts., 4, 173-181 (1983).

13) D. M. J. Dickson and G. O. Kirst: New Phytol., 106, 657-666 (1987).

14) K. Nakajima, A. Uchidd, and Y. Ihida: Mem. Koshien Univ., 16A, 7-10 (1988).

15) K. Nakajima, A. Uchida, and $Y$. Ishida: Nippon Suisan Gakkaishi, 55, 689-695 (1989).

16) K. Nakajima, A. Uchida, and Y. Ishida: Nippon Suisan Gakkaishi, 55, 1291 (1989).

17) K. Nakajima, A. Uchida, and Y. Ishida: Nippon Suisan Gakkaishi, 56, 1151-1154 (1990).

18) J. Van. Diggelin, J. Rozema, D. M. J. Dickson, and R. Broekman: New Phytol., 103, 573-586 (1986).

19) A. Chrominski, D. J. Weber, B. N. Smith, F. Heigerhorst, R. D. Horroks, and K.W. Burgener: Naturwissenschaften, 76, 473-475 (1989).

20) K. Nakajima: Mem. Koshien Univ., 17A, 1-8 (1990). 